OPEN ACCESS

Edited by: Madhuchhanda Bhattacharjee, University of Hyderabad, India

Reviewed by: Juan-Manuel Anaya, Rosario University, Colombia Tanima Bose, Ludwig Maximilian University of Munich, Germany

Carolina Ramirez-Santana,

Rosario University, Colombia

*Correspondence: Ruijie Zhang zhangrihmu@163.com

Fengmin Zhang fengminzhang@ems.hrbmu.edu.cn

Specialty section:

This article was submitted to Computational Genomics, a section of the journal

Frontiers in Genetics

Received: 13 August 2021 Accepted: 27 October 2021 Published: 11 November 2021

Citation:

Ding Y, Cui M, Qian J, Wang C, Shen $Q$, Ren H, Li L, Zhang $F$ and Zhang $R$ (2021) Calculation of Similarity Between 26 Autoimmune Diseases Based on Three Measurements Including Network, Function, and Semantics.

Front. Genet. 12:758041. doi: 10.3389/fgene.2021.758041

\section{Calculation of Similarity Between 26 Autoimmune Diseases Based on Three Measurements Including Network, Function, and Semantics}

\author{
Yanjun Ding ${ }^{1,2}$, Mintian Cui ${ }^{1}$, Jun Qian ${ }^{2}$, Chao Wang ${ }^{1}$, Qi Shen ${ }^{1}$, Hongbiao Ren ${ }^{1}$, \\ Liangshuang $\mathrm{Li}^{1}$, Fengmin Zhang $^{2 *}$ and Ruijie Zhang ${ }^{1 *}$
}

${ }^{1}$ College of Bioinformatics Science and Technology, Harbin Medical University, Harbin, China, ${ }^{2}$ Department of Microbiology, WU Lien-Teh Institute, Harbin Medical University, Harbin, China

Autoimmune diseases (ADs) are a broad range of diseases in which the immune response to self-antigens causes damage or disorder of tissues, and the genetic susceptibility is regarded as the key etiology of ADs. Accumulating evidence has suggested that there are certain commonalities among different ADs. However, the theoretical research about similarity between ADs is still limited. In this work, we first computed the genetic similarity between 26 ADs based on three measurements: network similarity (NetSim), functional similarity (FunSim), and semantic similarity (SemSim), and systematically identified three significant pairs of similar ADs: rheumatoid arthritis (RA) and systemic lupus erythematosus (SLE), myasthenia gravis (MG) and autoimmune thyroiditis (AIT), and autoimmune polyendocrinopathies (AP) and uveomeningoencephalitic syndrome (Vogt-Koyanagi-Harada syndrome, VKH). Then we investigated the gene ontology terms and pathways enriched by the three significant $A D$ pairs through functional analysis. By the cluster analysis on the similarity matrix of 26 ADs, we embedded the three significant $A D$ pairs in three different disease clusters respectively, and the ADs of each disease cluster might have high genetic similarity. We also detected the risk genes in common among the ADs which belonged to the same disease cluster. Overall, our findings will provide significant insight in the commonalities of different ADs in genetics, and contribute to the discovery of novel biomarkers and the development of new therapeutic methods for ADs.

Keywords: ADs, genetic susceptibility, network similarity, functional similarity, semantic similarity, autoimmune tautology

\section{INTRODUCTION}

Autoimmune diseases (ADs) are characterized by causing abnormal immune response which can damage human tissues as a result of the loss of immune tolerance to self-antigens (Margo and Harman, 2016). ADs affect more than 5\% of the global population, the incidence and mortality of which have also increased markedly (Ji et al., 2016). Possible causes contain genetic, environmental, hormonal, and immunological factors (Stojanovich and Marisavljevich, 2008). However, neither the inner mechanism action nor the etiology of ADs is clear and there is still no effective cure for these diseases (Rosenblum et al., 2012; Li et al., 2017). 
ADs share several clinical signs and symptoms, physiopathological mechanisms, and environmental and genetic factors, and this fact indicates that they have a common origin, which has been called the autoimmune tautology. A growing body of evidence has indicated the existence of the autoimmune tautology among various ADs: 1) Different ADs exhibit the same phenotypic characteristics (Anaya, 2017). These diseases, whether organ-specific or systematic, show tissue and organ damage and inflammatory pathological features (Place and Kanneganti, 2020). 2) Different ADs exhibit the same clinical characteristics. Clinically, the results from serological examinations of patients often overlap. And the same patient may suffer from two or more ADs simultaneously, which has been called the polyautoimmunity (PolyA) (Anaya, 2014). In addition, there is a tendency for ADs to cluster within families (Cardenas-Roldan et al., 2013). 3) Different ADs exhibit the same genetic characteristics. ADs are caused by the mutation of multiple loci in the human genome and share the same main genetic loci (Anaya et al., 2006). For example, a previous study indicated that the human leukocyte antigen (HLA) is a susceptibility gene shared by multiple ADs (Cruz-Tapias et al., 2012). And Ueda et al. (2003) found that a molecule encoded by CTLA4 was vital for negative regulation of the immune system and could enhance the risk of several ADs, such as Graves disease, autoimmune hypothyroidism, and type 1 diabetes mellitus (T1D), which indicated that ADs might share similar pathogenic mechanisms. Li et al. (2015) proved that different pediatric ADs shared the same genetic variation. They analyzed the clinical cases of ten different ADs and found many of these diseases were familial and the patients often suffered from several $\mathrm{ADs}$ at the same time. In this study, 27 significant risk genetic loci were identified, of which 22 were shared by at least two ADs and 19 loci were shared by at least three ADs. Thus, identification of risk genes shared by multiple ADs may help to explain the development of PolyA. 4) In addition, different $\mathrm{ADs}$ also exhibit the same epigenetic characteristics. Epigenetic researches found that ADs shared similar epigenetic mechanisms. For instance, the DNA promoter region in the target cells of systemic lupus erythematosus (SLE) and rheumatoid arthritis (RA) showed low methylation (Quintero-Ronderos and Montoya-Ortiz, 2012). The strong similarity among ADs provides us with a deeper understanding of the common underlying mechanisms of ADs, and also prompts researchers to classify ADs. Therefore, the studies on the genetic similarity of ADs can help us to dissect $\mathrm{AD}$ pathogenesis, and contribute to the discovery of novel biomarkers and the development of new therapeutic methods for ADs, which is extremely important in clinical research.

In this study, we utilized three measurements, including network similarity, functional similarity, and semantic similarity, to analyze genetic similarity between 26 ADs (the workflow diagram is shown in Figure 1). We identified three significant pairs of similar ADs by multi-step computational approaches. Besides, based on the similarity matrix of $26 \mathrm{ADs}$, we found some other ADs which were similar to significant pairs of similar ADs by cluster analysis. And the risk genes shared by the ADs which belonged to the same disease cluster could be promising biomarkers for ADs. Our findings provided a novel perspective to understand the commonalities of different ADs in genetics and would facilitate $\mathrm{AD}$ mechanism research.

\section{MATERIALS AND METHODS}

\section{Collection of AD Terms and AD-Related Genes}

The AD terms (category C20.111), including 68 diseases, were acquired from the Medical Subject Headings (MeSH, https:// www.nlm.nih.gov/mesh/meshhome.html). After removing the complications of ADs, we extracted human disease-related genes from the Genetic Association Database (GAD, https:// geneticassociationdb.nih.gov/) (Becker et al., 2004) and mapped these genes to the $\mathrm{AD}$ terms for integration. The disease gene sets consisted of 267 related genes of 26 ADs (Supplementary Table S1).

\section{Calculation of Network Similarity Between ADs}

We downloaded the information on protein-protein interactions of human genes from the Human Protein Reference Database (HPRD, http://www.hprd.org/) (Peri et al., 2003) and used Cytoscape software (v3.8.2) (Shannon et al., 2003) to construct a human protein-protein interaction network. The topological properties of $\mathrm{AD}$-related genes in this network were computed (Hidalgo et al., 2009; Chavali et al., 2010). The gene set of disease $d$ was defined as $G=\left\{g_{1}, g_{2}, g_{3}, \ldots, g_{i}, \ldots, g_{k}\right\}$. In order to assessed the network similarity between ADs, we first calculated the average topological properties of each $\mathrm{AD}$ in the network as follows:

$$
\begin{gathered}
R=\frac{\sum_{1 \leq i \leq k} r_{i}}{k}, C=\frac{\sum_{1 \leq i \leq k} c_{i}}{k}, B=\frac{\sum_{1 \leq i \leq k} b_{i}}{k}, S=\frac{\sum_{1 \leq i \leq k} s_{i}}{k}, \text { and } \\
H=\frac{\sum_{1 \leq i \leq k} h_{i}}{k}
\end{gathered}
$$

where $k$ represents the number of genes in $G, g_{i}$ is the $i$ th gene of $G$, $r_{i}$ is the degree of $g_{i}, c_{i}$ is the clustering coefficient of $g_{i}, b_{i}$ is the betweenness centrality of $g_{i}, s_{i}$ is the average shortest path length of $g_{i}, h_{i}$ is the neighborhood connectivity of $g_{i}, R$ is the degree of $d, C$ is the clustering coefficient of $d, B$ is the betweenness centrality of $d, S$ is the average shortest path length of $d$, and $H$ is the neighborhood connectivity of $d$. As shown in Figure $1, d_{1}$ and $d_{2}$ are two ADs from MeSH, and $G_{1}$ and $G_{2}$ are gene sets related to $d_{1}$ and $d_{2}$. The average topological properties of $d_{1}$ and $d_{2}$ were defined as vector $T_{1}=\left\{R_{1}, C_{1}, B_{1}, S_{1}, H_{1}\right\}$ and vector $T_{2}=\left\{R_{2}, C_{2}, B_{2}, S_{2}, H_{2}\right\}$, respectively. We defined the network similarity (NetSim) score between $d_{1}$ and $d_{2}$ as the Pearson correlation coefficient (PCC) calculated with $T_{1}$ and $T_{2}$. The formula that was used as follows:

$$
\rho_{T_{1}, T_{2}}=\frac{\operatorname{cov}\left(T_{1}, T_{2}\right)}{\sigma_{T_{1}}, \sigma_{T_{2}}}
$$

where $\operatorname{cov}\left(T_{1}, T_{2}\right)$ is the covariance of variables $T_{1}$ and $T_{2}, \sigma T_{1}$ and $\sigma T_{2}$ are the standard deviations for $T_{1}$ and $T_{2}$. 


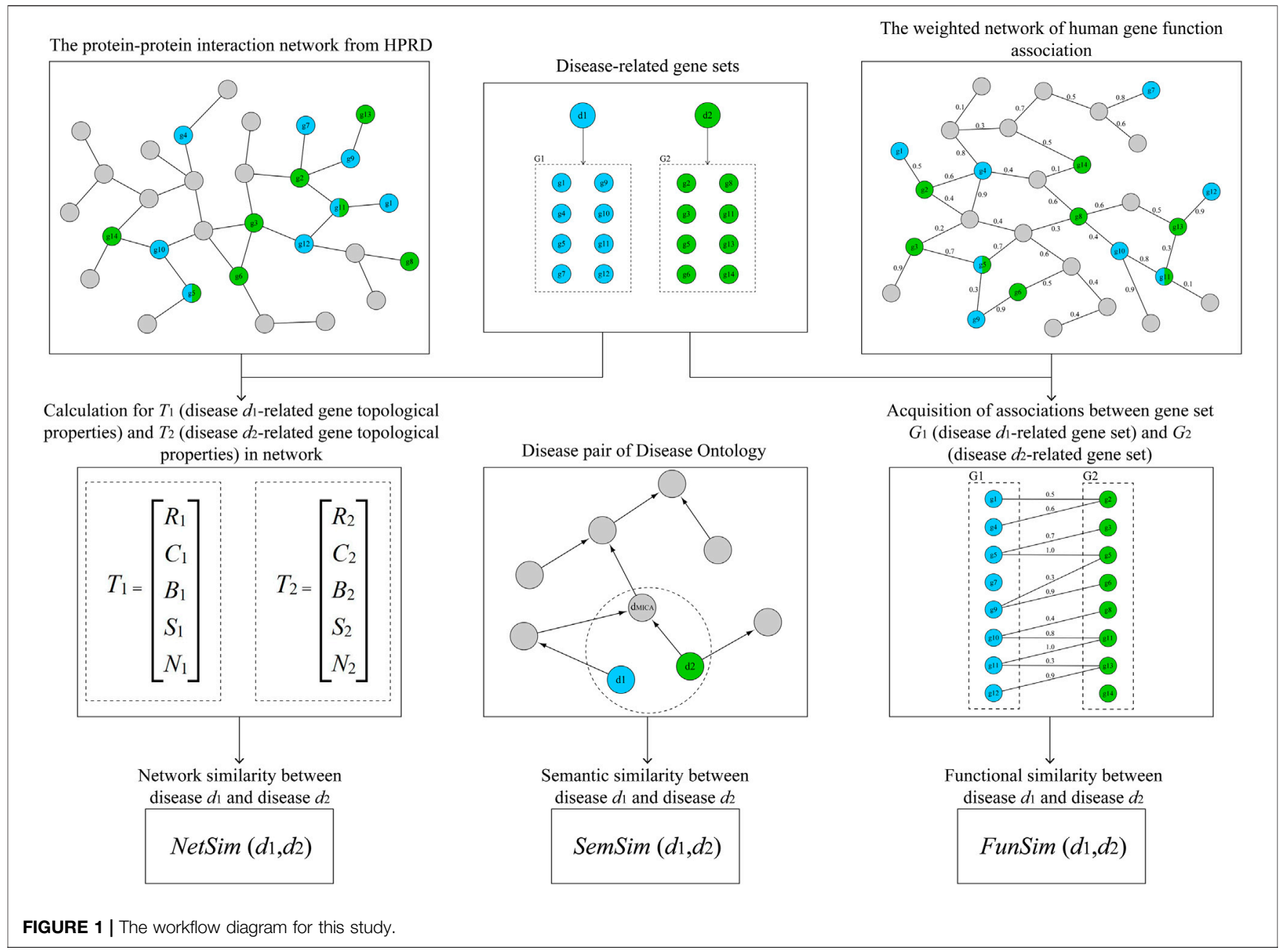

\section{Calculation of Functional Similarity Between ADs}

The data on the functional interactions of genes was downloaded from HumanNet, which is a human gene functional interaction network based on Gene Ontology annotation (Lee et al., 2011). Each interaction in HumanNet has a log likelihood score (LLS) that measures the probability of a functional association between genes (Cheng et al., 2014).

We downloaded LLSs between human genes from HumanNet and normalized the LLSs as follows:

$$
L L S_{N}\left(g_{i}, g_{j}\right)=\frac{L L S\left(g_{i}, g_{j}\right)-L L S_{\min }}{L L S_{\max }-L L S_{\min }}
$$

where $g_{i}$ and $g_{j}$ are the $i$ th and $j$ th gene respectively. $L L S_{N}\left(g_{i}, g_{j}\right)$ indicates LLS between $g_{i}$ and $g_{j}$ after normalization. $L L S\left(g_{i}, g_{j}\right)$ indicates LLS between $g_{i}$ and $g_{j}$. LLS min and $L L S_{\max }$ are the minimum LLS and the maximum LLS of HumanNet respectively.

The functional similarity (FunSim) score between a pair of genes was defined as follows:
$\operatorname{FunSim}\left(g_{i}, g_{j}\right)= \begin{cases}1 & i=j \\ L L S_{N} & i \neq j \text { and } e(i, j) \in \mathrm{E}(\text { HumanNet }) \\ 0 & i \neq j \text { and } e(i, j) \notin \mathrm{E}(\text { HumanNet })\end{cases}$

where $e(i, j)$ represents the interaction edge between $g_{i}$ and $g_{j}$. $\mathrm{E}$ (HumanNet) is a set including all the edges of HumanNet.

Next, we defined the functional association between a gene $g$ and a gene set $G=\left\{g_{1}, g_{2}, g_{3}, \ldots, g_{i}, \ldots, g_{k}\right\}$ as follows:

$$
F_{G}(g)=\max _{1 \leq i \leq k}\left(\operatorname{FunSim}\left(g, g_{i}\right)\right), g_{i} \in G
$$

where $k$ represents the number of genes in $G, g_{i}$ is the $i$ th gene of $G$.

$G_{1}=\left\{g_{11}, g_{12}, \ldots, g_{1 i}, \ldots, g_{1 m}\right\}$ and $G_{2}=\left\{g_{21}, g_{22}, \ldots, g_{2 j}, \ldots\right.$, $\left.g_{2 n}\right\}$ are gene sets related to $d_{1}$ and $d_{2}$ respectively. $m$ is the number of genes in $G_{1}$, and $n$ is the number of genes in $G_{2}$. We defined FunSim score of $d_{1}$ and $d_{2}$ as follows:

$\operatorname{FunSim}\left(d_{1}, d_{2}\right)=\frac{\sum_{1 \leq i \leq m} F_{G_{2}}\left(g_{1 i}\right)+\sum_{1 \leq j \leq n} F_{G_{1}}\left(g_{2 j}\right)}{m+n}, g_{1 i} \in G_{1}, g_{2 j} \in G_{2}$ 


\section{Calculation of Semantic Similarity Between ADs}

Semantic similarity is a method to measure the closeness between two terms, according to a given ontology (Zhang and Lai, 2015; Zhang and Lai, 2016; Del Prete et al., 2018). The Resnik method was applied to our study. The human disease terms were obtained from the Human Disease Ontology (DO, http://www.disease-ontology. org) (Schriml et al., 2019). The DO includes the breadth of common and rare diseases, organized as a directed acyclic graph in which a term represents a DO term and an edge represents an "IS_A" relationship between diseases. The information content (IC) of each DO term could be calculated as follows:

$$
I C(d)=-\log \left(\frac{n}{N}\right)
$$

where $d$ is a disease term of DO, $n$ is the number of genes related to $d$, and $N$ is the total number of genes related to DO. As shown in Figure $1, d_{1}$ and $d_{2}$ are two $\mathrm{AD}$ terms of $\mathrm{DO}$, and $d_{M I C A}$ is the most informative common ancestor (MICA) of $d_{1}$ and $d_{2}$. The MICA means the ancestor that has the maximum IC among all the common ancestors between terms of ontology. And we defined the semantic similarity (SemSim) score of $d_{1}$ and $d_{2}$ as follows:

$$
\operatorname{SemSim}\left(d_{1}, d_{2}\right)=I C\left(d_{M I C A}\right)
$$

\section{Calculation of Integrated Similarity Between ADs}

To identify more reliable pairs of similar ADs, we integrated above three kinds of similarity (NetSim, FunSim, and SemSim) scores to comprehensively determine the levels of similarity between $\mathrm{ADs}$ as follows:

$$
\operatorname{Integrated} \operatorname{Sim}\left(d_{1}, d_{2}\right)=\sqrt[3]{\operatorname{NetSim}\left(d_{1}, d_{2}\right) \cdot \operatorname{FunSim}\left(d_{1}, d_{2}\right) \cdot \operatorname{SemSim}\left(d_{1}, d_{2}\right)}
$$

where $d_{1}$ and $d_{2}$ are two $\mathrm{AD}$ terms of $\mathrm{MeSH}$.

\section{Functional Analysis of Related Genes of Similar ADs}

Functional enrichment analysis of Gene Ontology (GO) and Kyoto encyclopedia of genes and genomes (KEGG) for related genes of similar ADs was performed to infer potential biological processes and pathways using the DAVID Bioinformatics Tool (http://david. abcc.ncifcrf.gov/, version 6.7) (Huang Da et al., 2009). The $p$-values for the biological processes and pathways were adjusted for false discovery rate (FDR) by the Benjamini-Hochberg method. The biological processes and pathways with FDR less than 0.05 were considered statistically significant functional categories.

\section{Cluster Analysis for ADs Based on Three Kinds of Similarity}

To determine whether multiple ADs had high genetic similarity, hierarchical clustering was performed on the integrated similarity scores between 26 ADs based on the Euclidean distance. The $d_{1}$ and $d_{2}$ are two diseases of 26 ADs. We defined the Euclidean distance between $d_{1}$ and $d_{2}$ as follows:

$$
E\left(d_{1}, d_{2}\right)=\sqrt{\sum_{1 \leq i \leq 26}\left(x_{1 i}-x_{2 i}\right)^{2}}
$$

where $x_{1 i}$ is the integrated similarity score between $d_{1}$ and $i$ th disease of $26 \mathrm{ADs}, x_{2 i}$ is the integrated similarity score between $d_{2}$ and $i$ th disease of $26 \mathrm{ADs}$. The $26 \mathrm{ADs}$ were clustered based on the Euclidean distances between diseases and there were shorter Euclidean distances between ADs belonging to the same disease cluster.

\section{RESULTS}

\section{Identification of Potential Pairs of Similar ADs}

To identify pairs of similar diseases in genetics, we calculated similarity between 26 ADs based on three measurements. All of $\mathrm{AD}$ pairs were sorted in descending according to their scores of NetSim, FunSim, and SemSim. To enhance the reliability of the study, we selected ten $\mathrm{AD}$ pairs from intersection of top $50 \mathrm{AD}$ pairs with the highest NetSim score, top $50 \mathrm{AD}$ pairs with the highest FunSim score, and top $50 \mathrm{AD}$ pairs with the highest SemSim score for further analysis (Figure 2 and Supplementary Tables S2-4), which were considered as potential pairs of similar ADs.

Further, a network was generated on the ten $\mathrm{AD}$ pairs and their related genes, including $14 \mathrm{ADs}$ and 247 genes (Figure 3). The topological properties of the network were investigated. We extracted 35 genes each of whose degree was greater than or equal to three from the network as the possible $\mathrm{AD}$ relevant genes. The top five genes regarding degree were $H L A-D R B 1, H L A-D Q B 1$, CTLA4, HLA-DQA1, and TNF, indicating that these genes were critical in multiple ADs. Previous researches have demonstrated that these genes are associated with many ADs and exert various functions in human autoimmune disorders. Besides, we ascertained that RA and multiple sclerosis (MS) involved more similarity relationships than other $\mathrm{AD}$ in this network, implying that the pathogenesis of RA and MS might exist in most of ADs from the network. What's more, a pair of diseases with higher number of shared genes, suggesting they likely have higher genetic similarity. Thus, the ten potential pairs of similar ADs were ranked by their number of shared genes and shown in Table 1. And the top-rank disease pair is RA and SLE, followed by T1D and RA, and RA and MS.

\section{Functional Implication of the Genes Related to Multiple ADs}

To explore common genetic mechanisms of a variety of ADs, we performed functional enrichment analysis of GO and KEGG for the $35 \mathrm{AD}$ relevant genes (Figure 4A). The cutoff criterion was a FDR less than 0.05. The top ten significant GO terms in the BP were mainly associated with immune response, antigen processing and presentation, and interferon gamma (IFN $\gamma$ )related functions (Figure 4B). Notably, IFNG was involved in 


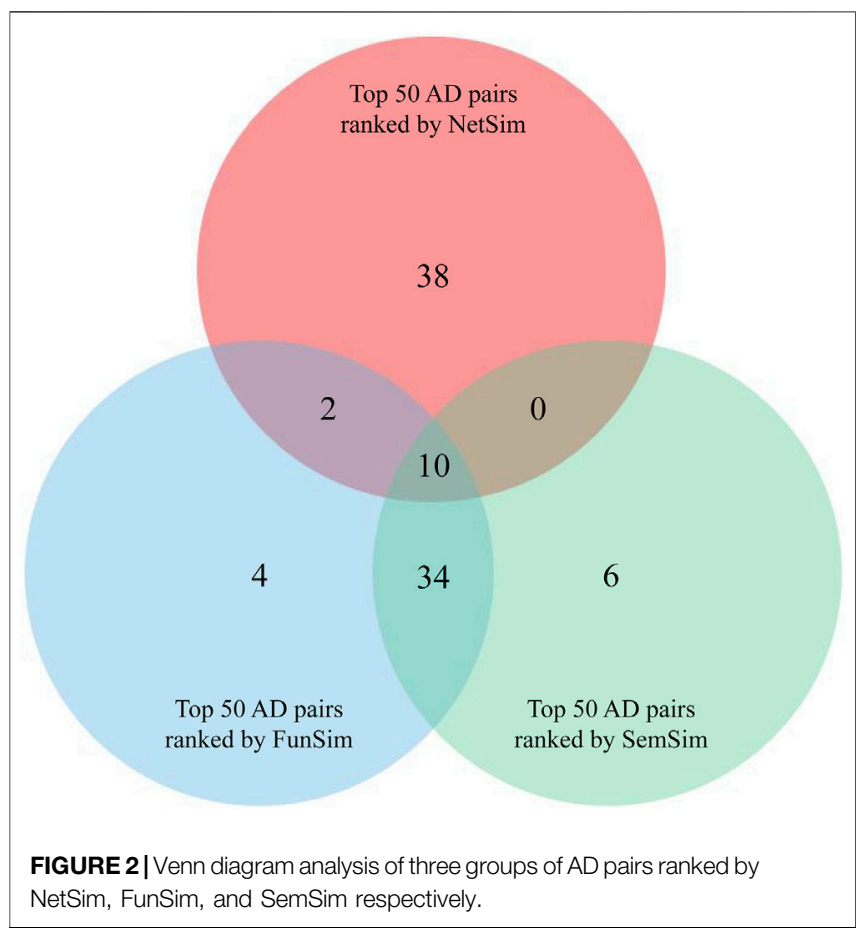

the top three GO terms and was defined as a hub gene. IFNG can encode IFN $\gamma$ that is a cytokine that is critical for innate and adaptive immunity against viral, bacterial, and protozoan infections. And aberrant IFN $\gamma$ expression is associated with a number of ADs, such as RA and SLE (Hu and Ivashkiv, 2009; Barrat et al., 2019). The top ten significant KEGG pathways contained four AD-correlated pathways, such as "inflammatory bowel disease (IBD)," "autoimmune thyroid disease," "type 1 diabetes mellitus," and "rheumatoid arthritis," which illustrated that the 35 genes might induce the initiation and development of multiple ADs (Figure 4C). HLA-DQB1, HLA-DRB1, HLA$D P B 1, H L A-D Q A 1$ were involved in all of the top ten KEGG pathways and were defined as hub genes. The four genes belonged to HLA class II alleles which were suggested to contribute to the susceptibility and resistance to ADs (Wieber et al., 2021).

\section{Identification of Significant Pairs of Similar ADs}

To identify more reliable pairs of similar ADs, we integrated the three measurements to compute integrated similarity scores between 26 ADs (see Materials and Methods). All of AD pairs were sorted in descending according to their integrated similarity scores. And the top ten $\mathrm{AD}$ pairs were extracted for further analysis (Table 2). We found that the ten $\mathrm{AD}$ pairs contained three potential pairs of similar ADs consisting of RA and SLE, myasthenia gravis (MG) and autoimmune thyroiditis (AIT), and autoimmune polyendocrinopathies (AP) and uveomeningoencephalitic syndrome (Vogt-Koyanagi-Harada syndrome, $\mathrm{VKH}$ ) which were defined as the significant pairs of similar ADs.

\section{Functional Analysis of Related Genes of Significant Pairs of Similar ADs}

To reveal the underlying mechanisms shared by two similar ADs, we performed functional enrichment analysis of related genes of RA and SLE, MG and AIT, and AP and VKH. The cutoff criterion was a FDR less than 0.05 . The related genes of RA and SLE were significantly enriched in GO terms mainly involved in immune response, inflammatory response, and IFN $\gamma$. The significant enriched pathways including RA, inflammatory bowel disease (IBD), tuberculosis, etc (Figures 5A,B). The related genes of $M G$ and AIT were mainly related to immune response (GO), antigen processing and presentation (GO), autoimmune thyroid disease (AITD) (KEGG), and allograft rejection (KEGG) (Figures 5C,D). Moreover, the related genes of $\mathrm{AP}$ and $\mathrm{VKH}$ were mainly associated with the antigen processing and presentation in GO and some pathways such as viral myocarditis, Staphylococcus aureus infection, AITD, intestinal immune network for IgA production, etc (Figures 5E,F).

\section{Hierarchical Clustering Result of 26 ADs Based on Three Similarity Measurements}

To determine whether there was high genetic similarity among multiple ADs, we applied hierarchical clustering to the integrated similarity matrix of 26 ADs. The disease clusters consisting of $\mathrm{AD}$ pairs with integrated similarity scores greater than 0.3 were considered to be significant. As shown in Figure 6A, three significant disease groups were identified from the $26 \mathrm{ADs}$, and the ADs of each disease group might have high genetic similarity. We found that the three significant pairs of similar ADs were located in three different clusters, respectively. In the cluster one, bullous pemphigoid might be similar to AP and VKH. The ADs of cluster one are involved in endocrine autoimmunity. For instance, bullous pemphigoid has been proved to be related to immunodysregulation polyendocrinopathy enteropathy X-linked syndrome (Mcginness et al., 2006). In the cluster two, pemphigus, Graves disease, Sjogren's syndrome, Addison Disease, and autoimmune hepatitis might be similar to MG and AIT. The ADs of cluster two contain the main AITDs (AIT and Graves disease) and frequent ADs involved in PolyA (AIT, Graves disease, and Sjogren's syndrome) (Amador-Patarroyo et al., 2012; Botello et al., 2020). In the cluster three, T1D and MS might be similar to RA and SLE. The ADs of cluster three are all chronic inflammatory ADs and share multiple genetic susceptibility loci (Richard-Miceli and Criswell, 2012). Then, we performed pathway enrichment analysis of the related genes of ADs of three significant clusters. The related genes of $\mathrm{ADs}$ of three disease clusters were significantly enriched in pathways of T1D and IBD (Figures 6B-D). Therefore, we infer that T1D and IBD can participate in PolyA in various AD patients.

Next, we detected the causal genes in common among the ADs which belonged to the same significant disease cluster, and these 


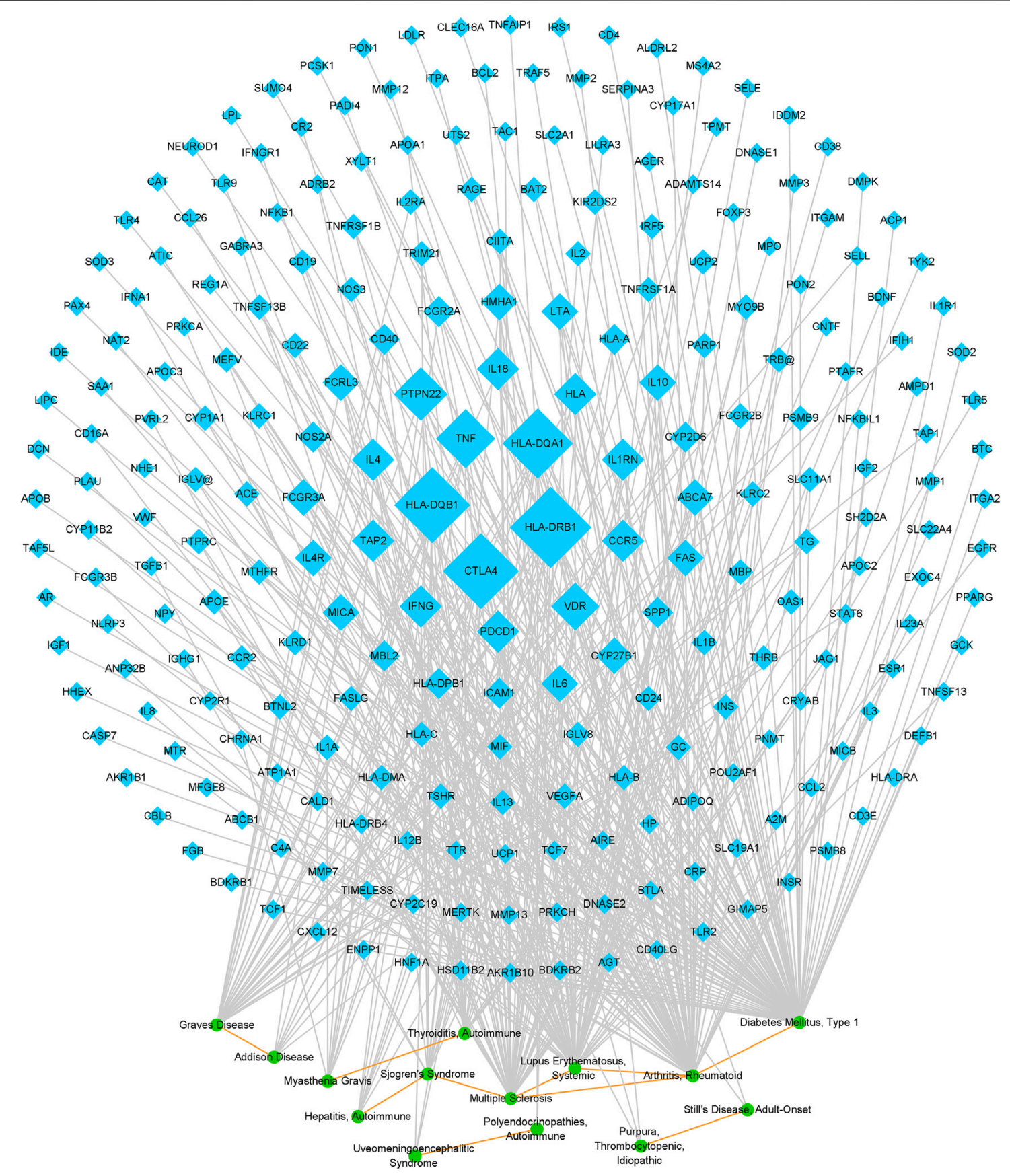

FIGURE 3 | Network on potential pairs of similar ADs and AD-gene relationships. The blue nodes represent genes, and the size of these nodes corresponds to the node degree. The green nodes represent ADs. The gray edges represent disease-gene relationships, and the orange edges represent potential AD similarity relationships.

genes could be used for PolyA research. As shown in Table 3, the $\mathrm{ADs}$ of cluster one shared one gene (HLA-DQA1); the ADs of cluster two shared two genes (HLA-DQB1 and $H L A-D R B 1)$; the ADs of cluster three shared eight genes (TNF, HLA-DRB1, PDCD1, PTPN22, CCR5, IL6, HLA-DQB1, and CTLA4). Identification of these genes will contribute to the discovery of novel prognostic, diagnostic, and therapeutic markers and justification of drug repurposing for ADs.

\section{DISCUSSION}

During the past years, numerous studies have confirmed that different ADs are similar in various aspects. Nevertheless, these studies just focused on several ADs, and lacking a comprehensive analysis on similarity between ADs from the perspective of genetics. To date, various disease similarity methods have been developed (Dozmorov, 2019). 
TABLE 1 | The ten potential pairs of similar ADs ranked by number of shared genes.

Rank

Autoimmune disease

Arthritis, Rheumatoid

Diabetes Mellitus, Type 1

Arthritis, Rheumatoid

Lupus Erythematosus, Systemic

Multiple Sclerosis

Myasthenia Gravis

Addison Disease

Hepatitis, Autoimmune

Polyendocrinopathies, Autoimmune

Purpura, Thrombocytopenic, Idiopathic
Autoimmune disease

Number

of shared gene

Lupus Erythematosus, Systemic

Arthritis, Rheumatoid

Multiple Sclerosis

Multiple Sclerosis

Sjogren's Syndrome

Thyroiditis, Autoimmune

Graves Disease

Sjogren's Syndrome

Uveomeningoencephalitic Syndrome

Still's Disease, Adult-Onset

\section{A}

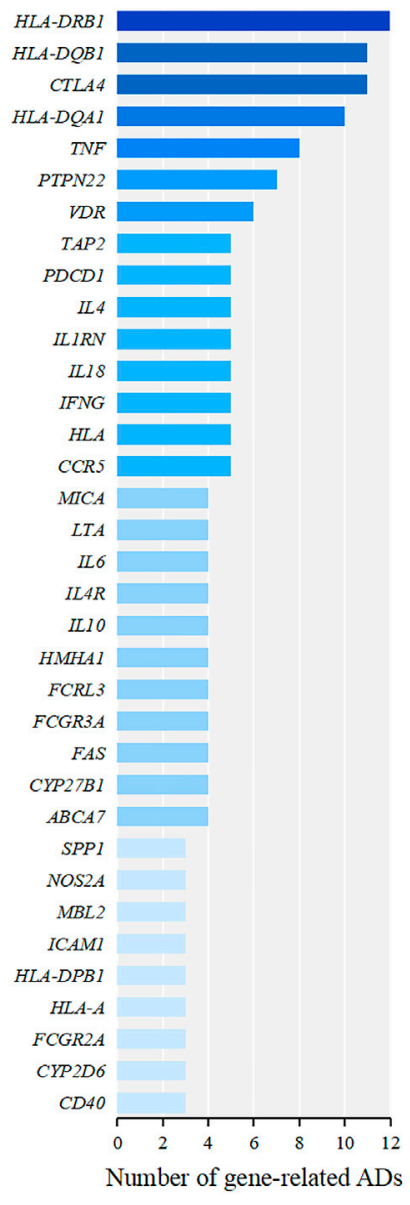

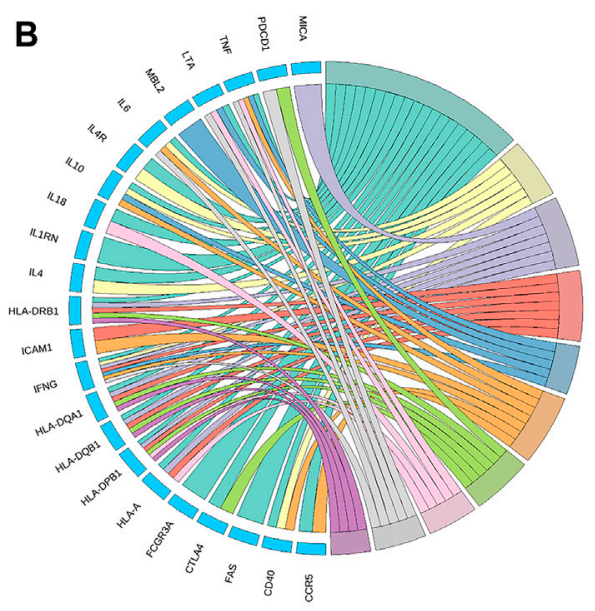
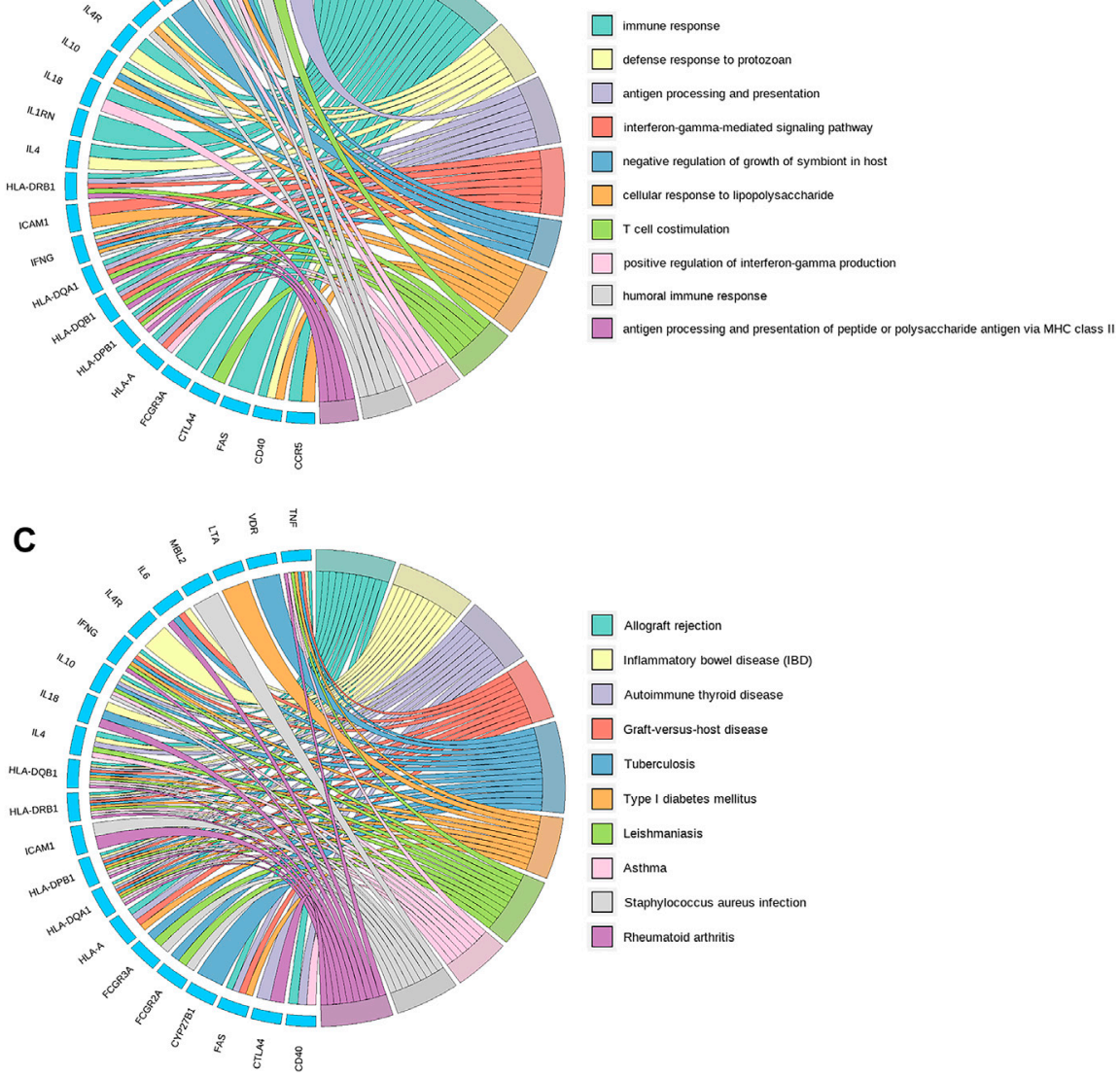

FIGURE 4 | Identification and functional enrichment analysis of AD relevant genes. (A) The genes correlated with at least three ADs derived from the AD similarity network, which are ranked by the number of related diseases. (B) Circos plot of top ten significant GO terms in the BP. (C) Circos plot of top ten significant KEGG pathways. The genes are displayed on the left half of the circos plots. The right half represents different GO terms or KEGG pathways with different colors. A gene is linked to a certain GO term or KEGG pathway by the colored bands.

In this study, we calculated the similarity scores between 26 ADs by means of three similarity measurements. To ensure the accuracy of the subsequent analysis, we combined the results of NetSim, FunSim, and SemSim to evaluate all the AD pairs. We found ten potential pairs of similar ADs that were utilized to form a network containing an overall insight of the information about $\mathrm{AD}-\mathrm{AD}$ relationships and $\mathrm{AD}$-gene relationships, which provided essential clues to understand the mechanisms shared by multiple ADs. Based on the AD pairs in this network, we detected three significant pairs of similar ADs (RA and SLE, MG and AIT, and AP and VKH), and then investigated the shared functional terms for each 
TABLE 2 | Top ten pairs of ADs ranked by integrated similarity scores.

\section{Rank}

Autoimmune disease

Polyendocrinopathies, Autoimmune

Thyroiditis, Autoimmune

Myasthenia Gravis

Addison Disease

Uveomeningoencephalitic Syndrome

Arthritis, Rheumatoid

Pemphigoid, Bullous

Hepatitis, Autoimmune

Anemia, Hemolytic, Autoimmune

Guillain-Barre Syndrome
Autoimmune disease

Integrated similarity score

Uveomeningoencephalitic Syndrome

0.704362256

0.605323256

0.540495323

0.53677382

0.534304723

0.527496589

0.52566119

0.507462033

0.499501

0.499448795

A

C interferon-gamma-mediated signaling pathway

T cell costimulation

immunoglobulin production involved in immunoglobulin mediated immune response humoral immune response mediated by circulating immunoglobulin

$\mathrm{T}$ cell receptor signaling pathway

detection of bacterium

antigen processing and presentation of exogenous peptide antigen via MHC class I

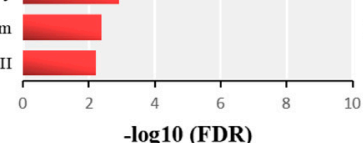

$-\log 10$ (FDR)

E

antigen processing and presentation of peptide or polysaccharide antigen via MHC class II antigen processing and presentation

$T$ cell costimulation

interferon-gamma-mediated signaling pathway

antigen processing and presentation of exogenous peptide antigen via MHC class II

$\mathrm{T}$ cell receptor signaling pathway

immunoglobulin production involved in immunoglobulin mediated immune response

immune response

humoral immune response mediated by circulating immunoglobulin

Enriched GO terms

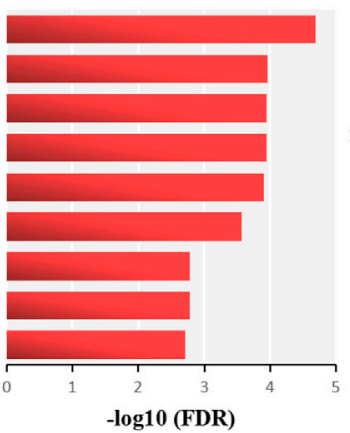

B

Enriched KEGG pathways

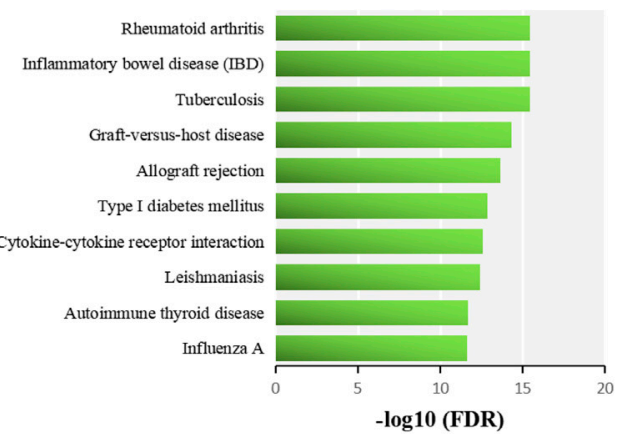

D

Enriched KEGG pathways

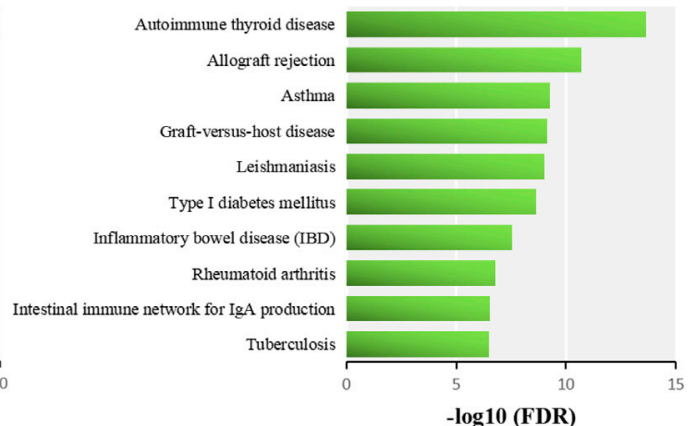

$\mathbf{F}$

Enriched KEGG pathways

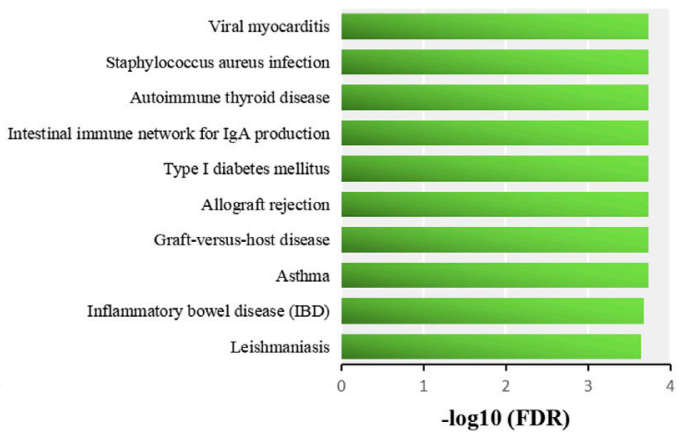

FIGURE 5 | Functional enrichment analysis of related genes of RA and SLE (A-B), MG and AIT (C-D), and AP and VKH (E-F). Enriched functional terms are sorted in descending order according to their-log10 (FDR), and the top ten significant GO terms in the BP and KEGG pathways of each significant pair of similar ADs are used for further analysis. 


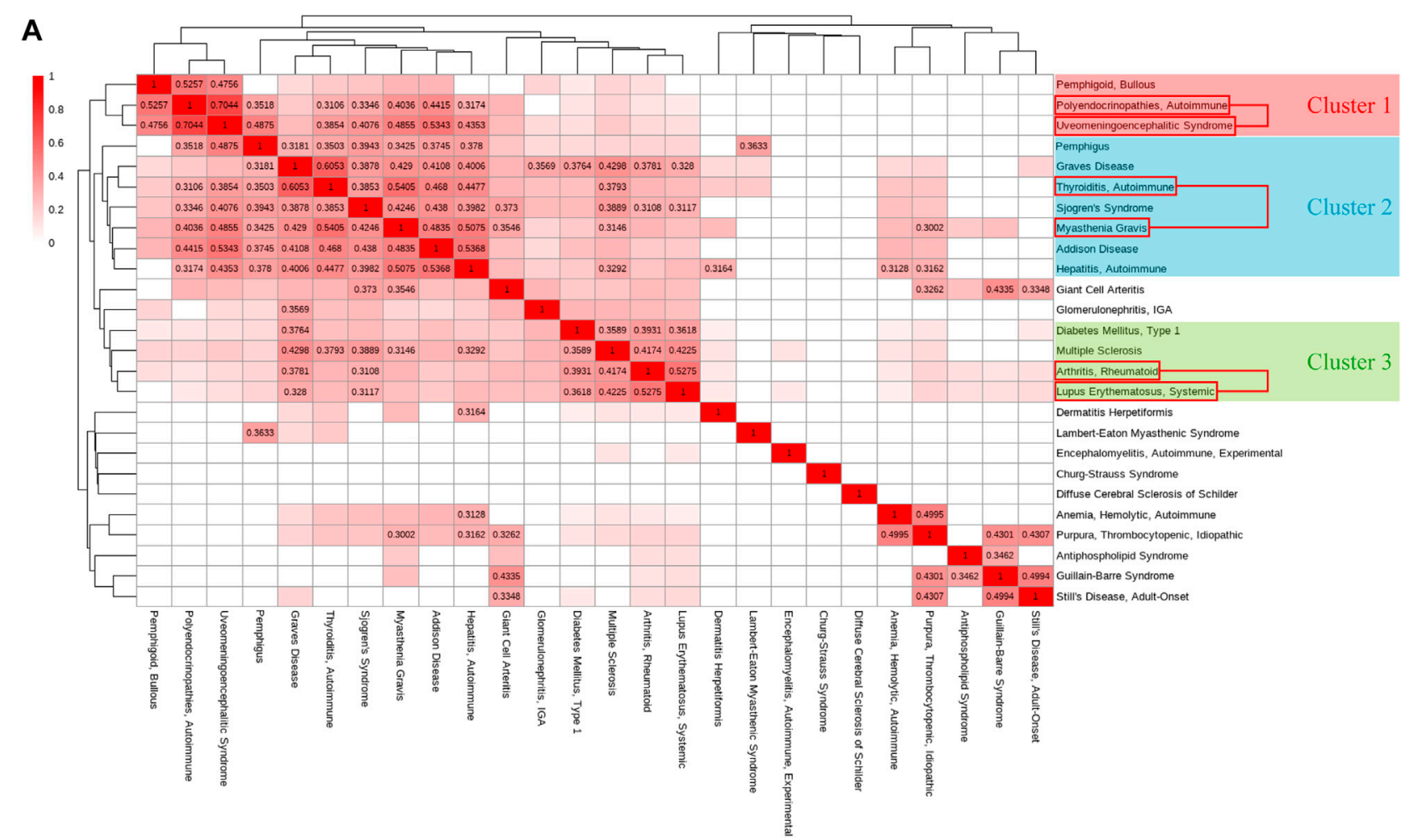

B

Enriched KEGG pathways

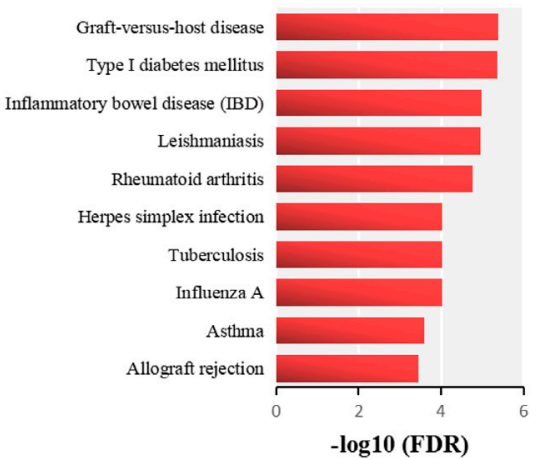

C

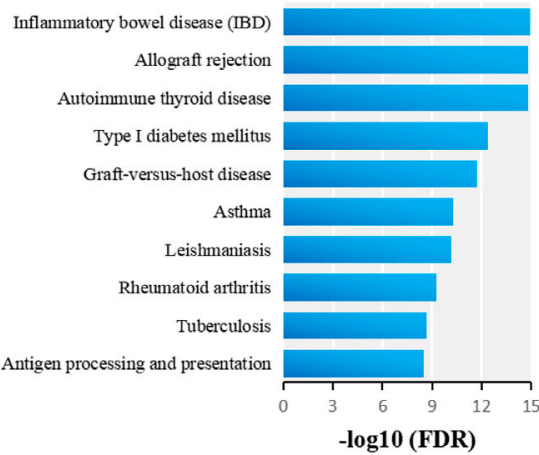

D Enriched KEGG pathways

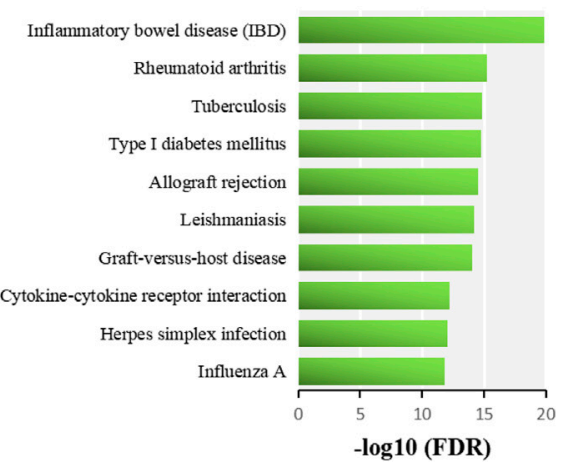

FIGURE 6 | Cluster analysis for 26 ADs and pathway enrichment analysis for ADs of each significant cluster. (A) The clustering heatmap illustrating the classification of 26 ADs based on Euclidean distances and integrated similarity scores between 26 ADs. The similarity values, which are greater than 0.3, are marked in the matrix. (B) The top ten significant KEGG pathways of related genes of ADs of cluster one ranked by-log10 (FDR). (C) The top ten significant KEGG pathways of related genes of ADs of cluster two ranked by-log10 (FDR). (D) The top ten significant KEGG pathways of related genes of ADs of cluster three ranked by-log10 (FDR).

significant AD pair. We also employed cluster analysis on the integrated similarity matrix of 26 ADs to acquire some other ADs which were similar to significant AD pairs in genetics, and identified the risk genes which belonged to the same disease cluster. These results still need to be verified by more studies, but we hope that our observations can help researchers to dissect the complex pathogenesis of ADs.

By the functional enrichment analysis of $35 \mathrm{AD}$ relevant genes, we mainly focused on GO terms involved in immune response, antigen processing and presentation, and IFN $\gamma$. The immune response is how the immune system defends against foreign invaders, such as bacteria or viruses (Chaplin, 2010). ADs are triggered by aberrant immune response which damages healthy body part and is influenced by a large number of genes (Hill et al., 2008; Gregersen and Olsson, 2009). We concluded that the 35 genes might trigger a variety of ADs. On the other hand, the dysfunction of antigen processing and presentation might influence the emergence of ADs (Ritz and Seliger, 2001). In human bodies, antigens are processed into peptides of a certain length in association with major histocompatibility complex (MHC) molecules. T cells are capable of recognizing these fragmented peptides bound to the MHC to initiate immune responses (Purcell et al., 2016; Kelly and Trowsdale, 2019; 
TABLE 3 | The shared causal genes among the ADs which belong to the same significant disease cluster.

\begin{tabular}{ll}
$\begin{array}{l}\text { Disease } \\
\text { cluster }\end{array}$ & \multicolumn{1}{c}{ Shared gene } \\
\hline $\begin{array}{l}\text { Cluster one } \\
\text { Cluster two }\end{array}$ & HLA-DQA-DQB1, HLA-DRB1 \\
Cluster three & TNF, HLA-DRB1, PDCD1, PTPN22, CCR5, IL6, HLA-DQB1, \\
& CTLA4
\end{tabular}

Italics refers to gene symbols (gene names).

Kotsias et al., 2019). As different ADs share the characteristic that risk is conferred by genes encoded within the MHC locus, antigen presentation generally seems to be crucial in ADs (Riedhammer and Weissert, 2015). For example, processing and presentation of selfantigens by different antigen presenting cells may result in MS (Stoeckle and Tolosa, 2010). Ultimately, IFN $\gamma$ is a pleiotropic cytokine secreted by immune cells and plays a critical role in innate and adaptive immunity (Tau and Rothman, 1999; Schoenborn and Wilson, 2007). Abnormal IFN $\gamma$ expression is correlated with considerable number of ADs. Although IFN $\gamma$ can mediate clearance of pathogenic insults, chronic exposure to IFN $\gamma$ is thought to cause many ADs, such as RA and SLE (Nielen et al., 2004; Lu et al., 2016). And the complex role of IFN $\gamma$ in ADs also has important therapeutic implications. Above evidences demonstrate that the three function aspects play important roles in $\mathrm{AD}$-related mechanisms.

With regard to the three significant pairs of similar ADs, several studies have confirmed these similarity relationships. For example, (Wang et al., 2020) found that familial RA, SLE, and primary Sjögren's syndrome shared common genetic characteristics, and the genetic variations in $\mathrm{T}$ cell receptor signaling pathway genes which might become novel molecular targets for therapeutic interventions for the three ADs. (Liu et al., 2019) found that $\mathrm{T}$ cell receptor could become a promising diagnostic marker for RA and SLE. In addition, previous studies have confirmed that MG and AIT are similar in many aspects (Marino et al., 1997; Lopomo and Berrih-Aknin, 2017), and AIT frequently accompanies MG (Mao et al., 2011; Kubiszewska et al., 2016). The two diseases are both organ-specific ADs with a clear pathogenic effect of antibodies. Meanwhile, MG and AIT share the same predisposing genes (such as PTPN22, CTLA4, and HLA) and pathological mechanisms (such as $\mathrm{T}$-cell immune-mediated mechanisms). Thus, we infer that $\mathrm{AD}$ genetic similarity research can help to explain the similar phenotypic and clinical features between ADs. These reports are consistent with our current results. Experimental studies on these $\mathrm{AD}$ pairs are desperately needed to provide important information to understand their intrinsic mechanisms. And further validation of these disease relationships in clinical trials will be a better option to turn them into clinical practice. Besides, the results of pathway enrichment analysis of related genes of significant pairs of similar ADs exposed possible PolyA. For example, the related genes of RA and SLE were enriched in pathways of IBD, T1D, and AITD. It was reported that AIT frequently coexisted with RA and SLE (OrdonezCanizares et al., 2020). Another study showed that AITD, RA, SLE, and IBD were observed in Sjögren's syndrome patients with PolyA (Amador-Patarroyo et al., 2012). And the related genes of MG and
AIT were enriched in pathways of T1D, IBD, and RA. Previous study found that the latent and overt PolyA in patients with AITD were associated with gastrointestinal, endocrinological, rheumatological, dermatological, and neurological ADs (Botello et al., 2020). The PolyA is not uncommon and multiple ADs that coexist in a single patient may share the same etiopathogenesis. Some genetic studies on $\mathrm{ADs}$ ignored the coexistence of other autoimmune conditions by implementing anachronistic nomenclature (i.e., primary or secondary ADs) (Rojas-Villarraga et al., 2012). We hope that researchers can take in account PolyA and concern whether or not patients have latent or overt PolyA in AD study.

With regard to the result of cluster analysis on $26 \mathrm{ADs}$, hitherto, a lot of reports have confirmed our viewpoint. For example, for the disease cluster two, a recent study found that chemokines were associated with the early phases of the autoimmune response in AIT, Graves disease, and Addison disease (Fallahi et al., 2020). For the disease cluster three, another study found major common gene expression changes at the target tissues of T1D, MS, RA, and SLE (Szymczak et al., 2021).

This study predicted AD pairs and clusters with high genetic similarity, as well as potential risk genes, biological processes, and pathways involved in multiple types of ADs. Despite the two diseases of a certain $\mathrm{AD}$ pair with high similarity score have different phenotypic or clinical features, they are likely to have similar or the same ways to elicit autoimmune responses in the human body. Consequently, we reason that similar ADs in genetics can be treated with similar therapeutics and drugs. We hope that these findings can aid in elucidating $\mathrm{AD}$ mechanisms, and provide more references for researchers.

\section{DATA AVAILABILITY STATEMENT}

The original contributions presented in the study are included in the article/Supplementary Material, further inquiries can be directed to the corresponding authors.

\section{AUTHOR CONTRIBUTIONS}

YD designed the study, analyzed the data, and drafted the manuscript. MC, JQ, and CW collected the data. QS, HR, and LL helped in analyzing the data, and calibrating the parameters of the algorithm. FZ and RZ provided ideas and revised the manuscript.

\section{FUNDING}

This work was supported by the Natural Science Foundation of Heilongjiang Province (Grant No. LH 2019C043).

\section{SUPPLEMENTARY MATERIAL}

The Supplementary Material for this article can be found online at: https://www.frontiersin.org/articles/10.3389/fgene.2021.758041/ full\#supplementary-material 


\section{REFERENCES}

Amador-Patarroyo, M. J., Arbelaez, J. G., Mantilla, R. D., Rodriguez-Rodriguez, A., Cárdenas-Roldán, J., Pineda-Tamayo, R., et al. (2012). Sjögren's Syndrome at the Crossroad of Polyautoimmunity. J. Autoimmun. 39, 199-205. doi:10.1016/ j.jaut.2012.05.008

Anaya, J.-M., Gómez, L., and Castiblanco, J. (2006). Is There a Common Genetic Basis for Autoimmune Diseases. Clin. Develop. Immunol. 13, 185-195. doi:10.1080/17402520600876762

Anaya, J.-M. (2017). The Autoimmune Tautology. A Summary of Evidence. Jt. Bone Spine 84, 251-253. doi:10.1016/j.jbspin.2016.11.012

Anaya, J.-M. (2014). The Diagnosis and Clinical Significance of Polyautoimmunity. Autoimmun. Rev. 13, 423-426. doi:10.1016/j.autrev.2014.01.049

Barrat, F. J., Crow, M. K., and Ivashkiv, L. B. (2019). Interferon Target-Gene Expression and Epigenomic Signatures in Health and Disease. Nat. Immunol. 20, 1574-1583. doi:10.1038/s41590-019-0466-2

Becker, K. G., Barnes, K. C., Bright, T. J., and Wang, S. A. (2004). The Genetic Association Database. Nat. Genet. 36, 431-432. doi:10.1038/ng0504-431

Botello, A., Herrán, M., Salcedo, V., Rodríguez, Y., Anaya, J. M., and Rojas, M. (2020). Prevalence of Latent and Overt Polyautoimmunity in Autoimmune Thyroid Disease: A Systematic Review and Meta-analysis. Clin. Endocrinol. 93, 375-389. doi:10.1111/cen.14304

Cárdenas-Roldán, J., Rojas-Villarraga, A., and Anaya, J.-M. (2013). How Do Autoimmune Diseases Cluster in Families? A Systematic Review and MetaAnalysis. BMC Med. 11, 73. doi:10.1186/1741-7015-11-73

Chaplin, D. D. (2010). Overview of the Immune Response. J. Allergy Clin. Immunol. 125, S3-S23. doi:10.1016/j.jaci.2009.12.980

Chavali, S., Barrenas, F., Kanduri, K., and Benson, M. (2010). Network Properties of Human Disease Genes with Pleiotropic Effects. BMC Syst. Biol. 4, 78. doi:10.1186/1752-0509-4-78

Cheng, L., Li, J., Ju, P., Peng, J., and Wang, Y. (2014). SemFunSim: a New Method for Measuring Disease Similarity by Integrating Semantic and Gene Functional Association. PLoS ONE 9, e99415. doi:10.1371/journal.pone.0099415

Cruz-Tapias, P., Pérez-Fernández, O. M., Rojas-Villarraga, A., RodríguezRodríguez, A., Arango, M.-T., and Anaya, J.-M. (2012). Shared HLA Class II in Six Autoimmune Diseases in Latin America: A Meta-Analysis. Autoimmune Dis. 2012, 1-10. doi:10.1155/2012/569728

Del Prete, E., Facchiano, A., and Liò, P. (2018). Bioinformatics Methodologies for Coeliac Disease and its Comorbidities. Brief. Bioinform. 21, 355-367. doi:10.1093/bib/bby109

Dozmorov, M. G. (2019). Disease Classification: from Phenotypic Similarity to Integrative Genomics and beyond. Brief. Bioinform. 20, 1769-1780. doi:10.1093/bib/bby049

Fallahi, P., Ferrari, S. M., Ragusa, F., Ruffilli, I., Elia, G., Paparo, S. R., et al. (2020). Th1 Chemokines in Autoimmune Endocrine Disorders. J. Clin. Endocrinol. Metab. 105, 1046-1060. doi:10.1210/clinem/dgz289

Gregersen, P. K., and Olsson, L. M. (2009). Recent Advances in the Genetics of Autoimmune Disease. Annu. Rev. Immunol. 27, 363-391. doi:10.1146/ annurev.immunol.021908.132653

Hidalgo, C. A., Blumm, N., Barabási, A.-L., and Christakis, N. A. (2009). A Dynamic Network Approach for the Study of Human Phenotypes. Plos Comput. Biol. 5, e1000353. doi:10.1371/journal.pcbi.1000353

Hill, N. J., King, C., and Flodstrom-Tullberg, M. (2008). Recent Acquisitions on the Genetic Basis of Autoimmune Disease. Front. Biosci. 13, 4838-4851. doi:10.2741/3043

$\mathrm{Hu}, \mathrm{X}$., and Ivashkiv, L. B. (2009). Cross-regulation of Signaling Pathways by Interferon- $\gamma$ : Implications for Immune Responses and Autoimmune Diseases. Immunity 31, 539-550. doi:10.1016/j.immuni.2009.09.002

Huang, D. W., Sherman, B. T., and Lempicki, R. A. (2009). Bioinformatics Enrichment Tools: Paths toward the Comprehensive Functional Analysis of Large Gene Lists. Nucleic Acids Res. 37, 1-13. doi:10.1093/nar/gkn923

Ji, J., Sundquist, J., and Sundquist, K. (2016). Gender-specific Incidence of Autoimmune Diseases from National Registers. J. Autoimmun. 69, 102-106. doi:10.1016/j.jaut.2016.03.003

Kelly, A., and Trowsdale, J. (2019). Genetics of Antigen Processing and Presentation. Immunogenetics 71, 161-170. doi:10.1007/s00251-018-1082-2
Kotsias, F., Cebrian, I., and Alloatti, A. (2019). Antigen Processing and Presentation. Int. Rev. Cel Mol. Biol. 348, 69-121. doi:10.1016/bs.ircmb.2019.07.005

Kubiszewska, J., Szyluk, B., Szczudlik, P., Bartoszewicz, Z., Dutkiewicz, M., Bielecki, M., et al. (2016). Prevalence and Impact of Autoimmune Thyroid Disease on Myasthenia Gravis Course. Brain Behav. 6, e00537. doi:10.1002/brb3.537

Lee, I., Blom, U. M., Wang, P. I., Shim, J. E., and Marcotte, E. M. (2011). Prioritizing Candidate Disease Genes by Network-Based Boosting of Genome-wide Association Data. Genome Res. 21, 1109-1121. doi:10.1101/gr.118992.110

Li, P., Zheng, Y., and Chen, X. (2017). Drugs for Autoimmune Inflammatory Diseases: From Small Molecule Compounds to Anti-TNF Biologics. Front. Pharmacol. 8, 460. doi:10.3389/fphar.2017.00460

Li, Y. R., Li, J., Zhao, S. D., Bradfield, J. P., Mentch, F. D., Maggadottir, S. M., et al. (2015). Meta-analysis of Shared Genetic Architecture across Ten Pediatric Autoimmune Diseases. Nat. Med. 21, 1018-1027. doi:10.1038/nm.3933

Liu, X., Zhang, W., Zhao, M., Fu, L., Liu, L., Wu, J., et al. (2019). T Cell Receptor $\beta$ Repertoires as Novel Diagnostic Markers for Systemic Lupus Erythematosus and Rheumatoid Arthritis. Ann. Rheum. Dis. 78, 1070-1078. doi:10.1136/ annrheumdis-2019-215442

Lopomo, A., and Berrih-Aknin, S. (2017). Autoimmune Thyroiditis and Myasthenia Gravis. Front. Endocrinol. 8, 169. doi:10.3389/fendo.2017.00169

Lu, R., Munroe, M. E., Guthridge, J. M., Bean, K. M., Fife, D. A., Chen, H., et al. (2016). Dysregulation of Innate and Adaptive Serum Mediators Precedes Systemic Lupus Erythematosus Classification and Improves Prognostic Accuracy of Autoantibodies. J. Autoimmun. 74, 182-193. doi:10.1016/j.jaut.2016.06.001

Mao, Z.-F., Yang, L.-X., Mo, X.-A., Qin, C., Lai, Y.-R., He, N.-Y., et al. (2011). Frequency of Autoimmune Diseases in Myasthenia Gravis: a Systematic Review. Int. J. Neurosci. 121, 121-129. doi:10.3109/00207454.2010.539307

Margo, C. E., and Harman, L. E. (2016). Autoimmune Disease: Conceptual History and Contributions of Ocular Immunology. Surv. Ophthalmol. 61, 680-688. doi:10.1016/j.survophthal.2016.04.006

Marinó, M., Ricciardi, R., Pinchera, A., Barbesino, G., Manetti, L., Chiovato, L., et al. (1997). Mild Clinical Expression of Myasthenia Gravis Associated with Autoimmune Thyroid Diseases1. J. Clin. Endocrinol. Metab. 82, 438-443. doi:10.1210/jcem.82.2.3749

McGinness, J. L., Bivens, M.-M. C., Greer, K. E., Patterson, J. W., and Saulsbury, F. T. (2006). Immune Dysregulation, Polyendocrinopathy, Enteropathy, X-Linked Syndrome (IPEX) Associated with Pemphigoid Nodularis: a Case Report and Review of the Literature. J. Am. Acad. Dermatol. 55, 143-148. doi:10.1016/ j.jaad.2005.08.047

Nielen, M. M. J., van Schaardenburg, D., Reesink, H. W., van de Stadt, R. J., van der Horst-Bruinsma, I. E., de Koning, M. H. M. T., et al. (2004). Specific Autoantibodies Precede the Symptoms of Rheumatoid Arthritis: a Study of Serial Measurements in Blood Donors. Arthritis Rheum. 50, 380-386. doi:10.1002/art.20018

Ordoñez-Cañizares, M. C., Mena-Vázquez, N., Redondo-Rodriguez, R., ManriqueArija, S., Jimenez-Núñez, F. G., Ureña-Garnica, I., et al. (2020). Frequency of Polyautoimmunity in Patients with Rheumatoid Arthritis and Systemic Lupus Erythematosus. J. Clin. Rheumatol. Publish Ahead of Print. doi:10.1097/ RHU.0000000000001574

Peri, S., Navarro, J. D., Amanchy, R., Kristiansen, T. Z., Jonnalagadda, C. K., Surendranath, V., et al. (2003). Development of Human Protein Reference Database as an Initial Platform for Approaching Systems Biology in Humans. Genome Res. 13, 2363-2371. doi:10.1101/gr.1680803

Place, D. E., and Kanneganti, T.-D. (2020). The Innate Immune System and Cell Death in Autoinflammatory and Autoimmune Disease. Curr. Opin. Immunol. 67, 95-105. doi:10.1016/j.coi.2020.10.013

Purcell, A. W., Croft, N. P., and Tscharke, D. C. (2016). Immunology by Numbers: Quantitation of Antigen Presentation Completes the Quantitative Milieu of Systems Immunology!. Curr. Opin. Immunol. 40, 88-95. doi:10.1016/ j.coi.2016.03.007

Quintero-Ronderos, P., and Montoya-Ortiz, G. (2012). Epigenetics and Autoimmune Diseases. Autoimmune Dis. 2012, 1-16. doi:10.1155/2012/593720

Richard-Miceli, C., and Criswell, L. A. (2012). Emerging Patterns of Genetic Overlap across Autoimmune Disorders. Genome Med. 4, 6. doi:10.1186/gm305

Riedhammer, C., and Weissert, R. (2015). Antigen Presentation, Autoantigens, and Immune Regulation in Multiple Sclerosis and Other Autoimmune Diseases. Front. Immunol. 6, 322. doi:10.3389/fimmu.2015.00322 
Ritz, U., and Seliger, B. (2001). The Transporter Associated with Antigen Processing (TAP): Structural Integrity, Expression, Function, and its Clinical Relevance. Mol. Med. 7, 149-158. doi:10.1007/bf03401948

Rojas-Villarraga, A., Amaya-Amaya, J., Rodriguez-Rodriguez, A., Mantilla, R. D., and Anaya, J.-M. (2012). Introducing Polyautoimmunity: Secondary Autoimmune Diseases No Longer Exist. Autoimmune Dis. 2012, 1-9. doi:10.1155/2012/254319

Rosenblum, M. D., Gratz, I. K., Paw, J. S., and Abbas, A. K. (2012). Treating Human Autoimmunity: Current Practice and Future Prospects. Sci. Transl. Med. 4, 125sr121. doi:10.1126/scitranslmed.3003504

Schoenborn, J. R., and Wilson, C. B. (2007). Regulation of Interferon- $\gamma$ during Innate and Adaptive Immune Responses. Adv. Immunol. 96, 41-101. doi:10.1016/S0065-2776(07)96002-2

Schriml, L. M., Mitraka, E., Munro, J., Tauber, B., Schor, M., Nickle, L., et al. (2019). Human Disease Ontology 2018 Update: Classification, Content and Workflow Expansion. Nucleic Acids Res. 47, D955-D962. doi:10.1093/nar/gky1032

Shannon, P., Markiel, A., Ozier, O., Baliga, N. S., Wang, J. T., Ramage, D., et al. (2003). Cytoscape: a Software Environment for Integrated Models of Biomolecular Interaction Networks. Genome Res. 13, 2498-2504. doi:10.1101/gr.1239303

Stoeckle, C., and Tolosa, E. (2009). Antigen Processing and Presentation in Multiple Sclerosis. Results Probl. Cel Differ. 51, 149-172. doi:10.1007/400_2009_22

Stojanovich, L., and Marisavljevich, D. (2008). Stress as a Trigger of Autoimmune Disease. Autoimmun. Rev. 7, 209-213. doi:10.1016/j.autrev.2007.11.007

Szymczak, F., Colli, M. L., Mamula, M. J., Evans-Molina, C., and Eizirik, D. L. (2021). Gene Expression Signatures of Target Tissues in Type 1 Diabetes, Lupus Erythematosus, Multiple Sclerosis, and Rheumatoid Arthritis. Sci. Adv. 7. doi:10.1126/sciadv.abd7600

Tau, G., and Rothman, P. (1999). Biologic Functions of the IFN-Gamma Receptors. Allergy 54, 1233-1251. doi:10.1034/j.1398-9995.1999.00099.x

Ueda, H., Howson, J. M. M., Esposito, L., Heward, J., Snook, H., Chamberlain, G., et al. (2003). Association of the T-Cell Regulatory Gene CTLA4 with Susceptibility to Autoimmune Disease. Nature 423, 506-511. doi:10.1038/nature01621
Wang, Y., Chen, S., Chen, J., Xie, X., Gao, S., Zhang, C., et al. (2020). Germline Genetic Patterns Underlying Familial Rheumatoid Arthritis, Systemic Lupus Erythematosus and Primary Sjögren's Syndrome Highlight T Cell-Initiated Autoimmunity. Ann. Rheum. Dis. 79, 268-275. doi:10.1136/annrheumdis2019-215533

Wieber, K., Zimmer, C. L., and Hertl, M. (2021). Detection of Autoreactive CD4+ T Cells by MHC Class II Multimers in HLA-Linked Human Autoimmune Diseases. J. Clin. Invest. 131 (9), e148674. doi:10.1172/JCI148674

Zhang, S.-B., and Lai, J.-H. (2016). Exploring Information from the Topology beneath the Gene Ontology Terms to Improve Semantic Similarity Measures. Gene 586, 148-157. doi:10.1016/j.gene.2016.04.024

Zhang, S.-B., and Lai, J.-H. (2015). Semantic Similarity Measurement between Gene Ontology Terms Based on Exclusively Inherited Shared Information. Gene 558, 108-117. doi:10.1016/j.gene.2014.12.062

Conflict of Interest: The authors declare that the research was conducted in the absence of any commercial or financial relationships that could be construed as a potential conflict of interest.

Publisher's Note: All claims expressed in this article are solely those of the authors and do not necessarily represent those of their affiliated organizations, or those of the publisher, the editors and the reviewers. Any product that may be evaluated in this article, or claim that may be made by its manufacturer, is not guaranteed or endorsed by the publisher.

Copyright (c) 2021 Ding, Cui, Qian, Wang, Shen, Ren, Li, Zhang and Zhang. This is an open-access article distributed under the terms of the Creative Commons Attribution License (CC BY). The use, distribution or reproduction in other forums is permitted, provided the original author(s) and the copyright owner(s) are credited and that the original publication in this journal is cited, in accordance with accepted academic practice. No use, distribution or reproduction is permitted which does not comply with these terms. 